\title{
Efficacy of a Synbiotic BIFILAC: Questionable study
}

Sir,

A recently reported "Randomized double blind controlled trial to evaluate the efficacy and safety of BIfilac in patients with acute viral diarrhea"1 has many flaws. First of all the author is not keen in letting the readers know what does bifilac contain. Is it a symbiotic or probiotic? What probiotic genera or specie was he studying or was it a combination of probiotics? There is an attempt to concentrate on the trade (product) name.

In the methodology there is no mention on what was used as a placebo and how was the blinding done (identical packaging). The type of randomization done has neither been mentioned nor the sample size calculation has been mentioned. We do not know which of these is the primary outcome measure as both frequency and duration of diarrhea have been listed. Hence the interpretation of the results is not possible and how do we know if the study has the power to predict the effect on these variables since it isn't mentioned. In the presentation of results apart from the primary outcome measures a flurry of other variables and end number of figures leave the reader confused and may be wrongly influenced. This study was not planned to see the effect on any of these variables (ORS and IVF administration etc).

\section{Author's Reply}

Sir,

1. The correspondent comments that whether Bifilac is a synbiotic or probiotic is not mentioned in the article. In discussion and conclusion (abstract as well as main article) it is mentioned that Bifilac is synbiotic.

\section{Bifilac ${ }^{\mathrm{TM}}$ (each $0.5 \mathrm{gm}$ sachet) contains}

Prebiotics: streptococcus faecalis T-110 30 million Clostridium butyricum TO-A 2 million Bacillus mesentricus TO-A 1 million

Probiotic : lactobacillus sporogenes 50 million

3. Trade name Bifilac is used in this article as this was a clinical drug trial using Bifilac as the synbiotic. This was a randomized double blinded controlled trial hence there is little chance of trial being bias to promote bifilac product as alleged by the correspondent.

4. In the discussion section the placebo used is mentioned as micro crystalline cellulose

5. BLINDING METHOD: In the methodology it is been mentioned Bifilac or placebo were administered in the form of sachets at a dose of 1 sachet three times daily up to 14 days.

Both Bifilac and placebo were dispensed in identical sachets, reconstituted similarly with $20 \mathrm{ml}$ of water and administered orally by identical schedule that is one sachet thrice daily up to 14 days.

6.Type of randomization : randomized to either control or intervention group by block randomization technique.

7.Sample size calculation was done according to statistical significance requirement. 80 children were enrolled to receive treatment with Bifilac or placebo in ratio of 1:1 thus gave rise to
Finally it is the first time I have seen an article which doesn't discuss the results of the present study with the available literature or theorises the effect seen. The author straight away decides the efficacy and safety of BIfilac and concludes. There is no declaration of competitive interest and I would be interested in knowing the name of the funding agency for this study. Was this a peer reviewed article? What is this category of special article? Aren't they peer reviewed?

Seema Alam

Consultant Incharge, Pediatric Gastroenterology Section

Reader, Department of Pediatrics, J. N. Medical College, AMU, Aligarh, Uttar Pradesh. India. Email seema_alam@hotmail.com,

[DOI-10.1007/s12098-009-0114-1]

\section{REFERENCE}

1. Narayanappa D. Randomized double blinded controlled trial to evaluate the efficacy and safety of Bifilac in patients with acute viral diarrhea. Indian J Pediatr 2008; 75:709-713.

40 patients in each treatment group.

8. Efficacy of bifilac in decreasing both frequency and duration of diarrhea were the primary objectives. Fig. 1 , table 1 and $1 \mathrm{~A}$ shows the comparative data of the mean frequency of diarrhea between Bifilac and placebo group, difference was statistically significant in favour of bifilac group. Fig 2 and table 2 show the comparative data of the mean duration of diarrhea between Bifilac and placebo group, difference was statistically significant in favour of bifilac group.

9.The correspondent comments that the study was not planned to see the effect on any of the other variables(ORS and IVF). This is not correct.

Secondary objective of the study was to evaluate the efficacy of bifilac in ameliorating the associated symptoms like dehydration (mean duration of ORS and IVF administration reflects duration of dehydration) and duration of rotaviral shedding.

Figs 3, 3A, 4, 4A, Tables 3, 3A ,4 ,4A ,5 show the comparative results on different variables and the difference between the two groups is statistically significant in favour of bifilac group. There is no reason to get confused if the reader goes through the article thoroughly.

10. There is ample evidence that probiotics reduce duration and severity of diarrhea-few of the studies have been quoted in the introduction part with references and mechanism theory is mentioned in brief at the beginning of introduction.

11. Competitive interests: none

12. Funding: Jhaver Research Foundation Ltd, Jhaver Centre, $4^{\text {th }}$ floor, 72, Marshalls Road, Egmore, Chennai 600008

D. Narayanappa, MD

Professor and Head, Pediatrics, J.S.S. Medical College Hospital, Ramanuja Road, Mysore, India E-mail: sinchabhi@yahoo.com 